XII.-Conversion of Ortho- into Para-, and of Parainto Ortho-quinone Derivatives.* I. The Condensation of Aldehydes with $\beta$-Hydroxy-a-naphthaquinone.

By Samuel C. Hooker and Willam C. Carxell.

Is the course of researches in the lapachol group, already partially communicated by one of us to the Society, numerous compounds of the general formula

I.<smiles>CC(C)(Br)C(O)OC1CCCCC1C(=O)O</smiles>

have been isolated, from which dehrdrating agents, preferably dilute or concentrated mineral acids, very readily remove water or hydrogen

* Compare Trans., 1893, 63, foot note p. 431; also Proc., 1893, 13. 
chloride or bromide, quantitatively converting them, without exception, into internal anhydrides.

It was at first supposed that the change was a simple one, involving nothing further than the elimination of water; but it was subsequently found that several of the lapachols, namely, chlorhydrolapachol, hydroxyhydrolapachol, and brombydroxyhydrolapachol, each gare two isomeric anhydrides, which proved to be related as follows.

II.<smiles>O=C1OC2OC3CCCCC1OC3O2</smiles>

III.<smiles>O=C1OOC2CCCCC1OCCO2</smiles>

Corresponding or a-anhydride.

Pseudo- or $\boldsymbol{\beta}$-anhydride.

In spite of the very varied conditions under which they have been exposed in contact with mineral acids, the remaining lapachols have given in each case only one anbydride, and these bave all proved to be $\beta$-naphthaquinone derivatives.

It can, therefore, be generally stated, at least so far as the compounds at present known in the lapachol group are concerned, that substances of the general formula I are more readily converted by the action of mineral acids into the $\beta$ - than into the $\alpha$-anhydrides, a change which involves the conversion of the para- into the ortho. quinone group. In order to ascertain how far this formation of the $\beta$-anhydrides would prove general, we have investigated the behaviour of compounds prepared synthetically from $\beta$-hydroxy- $x$-napbthaquinone.

Zincke and Thelen (Ber., 21, 2203) first observed that benzaldehyde and $\beta$-hydroxy- $\alpha$-naphthaquinone interact readily, and correctly attributed to the resulting compound the formula.

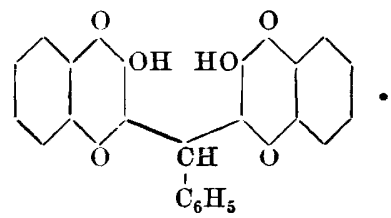

Schoch*, one of Zincke's pupils, subsequently extended the study of this reaction to other aldehydes, and prepared a number of compounds having the general formula

* "Ueber die Einwirkung ron Aldehyden und Ketonen auf Oxynaphtochinon," Inaugural Dissertat" in, Marburg, 1838. We are indebted to Professor Zincke for a copy of this pamphlet. 


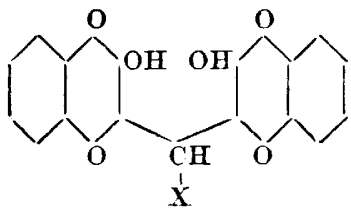

In preparing some of these compounds, small quantities of anhydrides were obtained as secondary products, to which the general formula

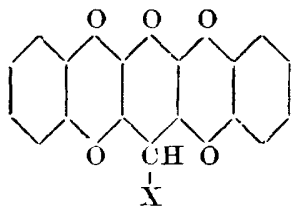

was assigned. These anhydrides were also in some cases directly obtained from the corresponding hydroxyl compounds by the action of dehydrating agents; but in every instance Schoch records that the yield was very small.

The behaviour of the lapachols in forming, as a rule, $\beta$-anhydrides in preference to $\alpha$-anhydrides, rendered questionable the structure attributed by Zincke and his pupils to the anhydrides obtained by them; and our experiments have conclusively prored that these compounds, or at least all that we have examined, are, in reality, as we anticipated, derivatives of $\beta$-naphthaquinone, to which one or other of the following formulæ must be attributed.

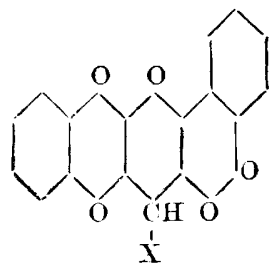

e $\beta$-Anlyydride.

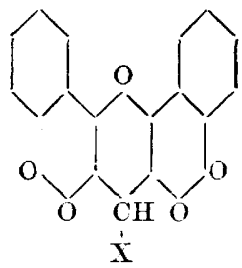

$\beta \beta$-Anlydride.

The anhydrides may be prepared quantitatively, or almost so, by the methods successfully used in the lapachol group. In no instance, however, in spite of many experiments, have we been successful in obtaining more than one anhydride from each compound.

All the anhydrides studied condense readily with orthotoluylenediamine, giving characteristic azines, an action which, in this case, can only be explained by the presence of the orthoquinone group. Up to the present time, the azines obtained have resulted from the action of one molecule or the diamine on one of the anhydride, so that, of the abore formulæ, the first, which is that of an $x p$-anhydride 
still containing one paraquinone group, is perbaps the more probable, although we do not regard the second as definitely excluded.

As a further proof of the presence of the orthoquinone group, it may be urged that there is a marked difference in the colour of the hydroxyl compounds, and of their corresponding anhydrides. The former, like $\alpha$-naphthaquinone, are yellow; the latter, like $\beta$-naphthaquinone, are orange or orange-red. A very considerable number of compounds of the lapachol group have been studied, and this change in colonr from yellow to orange or red has proved an unfailing indication of a simultaneous change from the para- to the orthoquinone.

It has been shown in the lapachol group, that both the $\alpha$ - and $\beta$-anhydrides take up water when boiled with dilute alkalis, yielding the same hydroxylapachol-a change, which, in the case of the $\beta$-anhydrides, involves the reconversion of the ortho- into the paraquinone.
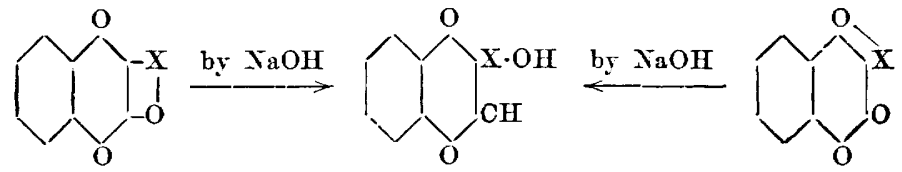

Precisely the same change occurs in the case of the synthesised $\beta$-anhydrides described in this paper only; it is more difficult to demonstrate, as the hydroxyl compounds examined very rapidly undergo change in contact even with very dilute caustic soda, a reaction which appears full of interest and will be stadied in detail by one of us.

Benzylidenedihydroxynaplthaquinone,

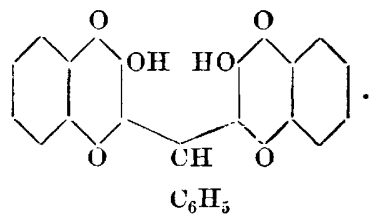

This compound was first obtained by Zincke and Thelen (Ber., 21 2203), and subsequentiy more fully examined by Schoch (Diss., Marburg, 1888, p. 11). In preparing the compound for our experiments, the directions of Zincke and Thelen were only partially followed. 8 grams of benzaldehyde, 8 grams of hydroxynaphthaquinone, and 24 c.c. of alcohol were leated in a corked flask on a water bath for about 45 minutes." The heating was then discontinued, and the solution diluted with 160 c.c. of alcohol ; the componnd

* Zincke and Thelen, also Schoch, recommend heating for sereral hours; this is unnecessary, as the action is complete in 45 minutes, and if the heating is extended over a longer period, a portion of the compound is converted into its anhydride. 
shortly afterwards commenced to separate in minute, yellow tufts of microscopic needles, which, after some hours, were collected and washed with alcohol. A microscopic examination revealed the presence, in small quantity, of orange-red needles, * which usually accompany to a somewhat greater extent the additional substance obtained on concentrating the mother liquor. The red needles are left for the most part undissolved when the crude substance is recrystallised from alcohol, but it is necessary to repeat the operation, sometimes more than once, in order that the separation may be rendered complete.

We found the melting point of benzylidenedihydroxynaphthaquinone to be about $230^{\circ}$ when rapidly heated, but as the substance decomposes when heated for a short time at temperatures slightly below this, its point of fusion is influenced by variations in the rate of heating.

Benzylidenedihydroxynaphthaquinone gives two distinct sodium derivatives, according as the bydrogen of one or both hydroxyl groups is displaced. The monosodium derivative crystallises in orange needles, and may be readily obtained by cautiously adding a 1 per cent. aqueous solution of sodium hydroxide to a boiling and nearly saturated alcoholic solution of the compound. The addition of the alkali is stopped as soon as the colour of the solution, which is at first orange, becomes permanently slightly carmine, and a precipitate of the carmine or normal salt begins to form. Small quantities of benzylidenedihydroxynaphthaquinone are then gradually added to the boiling solution until the carmine precipitate redissolves. On cooling, the solution first becomes turbid, and shortly afterwards orange needles of the sodium compound form throughout its entire volume. The normal salt, previously obtained by Zincke and Thelen, is a dark carmine-red, crystalline powder. When seen under the microscope, each grain appears as an almost perfectly formed octahedron. The very slight solubility of the salt, together with its very characteristic appearance and crystalline form, render it possible to identify minute quantities of benzylidevedihydroxynaphthaquinone with ease and certainty. It is simply necessary to dissolve the compound in a few drops of hot alcobol, aud add a drop or so of dilute aqueous solution of sodium hydroxide; if the alcoholic solution be a concentrated one, an abundant precipitate will be almost

* This substance, which appears to kave escaped the observation of Zincke, Thelen, and Schoch, differs essentially from the anhydride to be presently described : it dissolves in concentrated sulphuric acid, forming an intensely bluish-green solution, whereas the anhydride under the same conditions gives a reddish-brown solution. We made many unsuccessful attempts to obtain the compound in larger quantities. 
instantly formed, which should then be examined with a high powerin order that its crystalline form may be recognised.

$\alpha \beta$-Anhydride of Benzylidenedihydroxynaphthaquinone.-This compound is formed in small quantity in preparing benzylidenedihydroxynaphthaquinone if the time of digestion recommended above be much prolonged; it was in this way that Zincke and Thelen obtained it (Ber., 21, 2203); Scboch found that it was produced when an alcoholic solution of the hydroxyl compound was heated with a little sulphuric acid; but even in this case Schoch states that only small quantities were formed (Diss., Marburg, 1888, p. 14).

We have found that the compound can be prepared practically quantitatively in the following manner: -4 grams of benzylidenedihydroxynaph thaquinone are dissolved in 50 c.c. of boiling acetic acid, to which 2.5 c.c. of concentrated hydrochloric acid are then very gradually added; on continuing the boiling for a few minutes, a heavy precipitate of small, orange-red crystals rapidly forms, and may be collected when the solution has cooled. Wheu slightly washed witl. acetic acid, it is obtained in a condition pure enough for all ordinary purposes. A microscopic examination of the crystals revealed detached, well-formed, acicular prisms. The compound was purified for analysis by recrystallisation from acetic acid.

$0 \cdot 1837$ gave $0.5203 \mathrm{CO}_{2}$ and $0.585 \mathrm{H}_{2} \mathrm{O} . \quad \mathrm{C}=77 \cdot 24 ; \mathrm{H}=3.53$. $\mathrm{C}_{27} \mathrm{H}_{14} \mathrm{O}_{5}$ requires $\mathrm{C}=77.5 \mathrm{I} ; \mathrm{H}=3.35$ per cent.

When heated in a capillary tube, it commences to darken at about $245^{\circ}$. It dissolves with great difficulty in the ordinary solvents, being almost insoluble in ether, and only very slightly soluble in alcohol, rather more so in chloroform, benzene, and acetic acid.

The anhydride is reconverted into benzylidenedihydroxynaphthaquinone by boiling alkalis. A small quantity was moistened with aqueous 1 per cent. sodium hydroxide, thoroughly ground into a paste; more of the alkaline solution was then added, and the whole boiled for a few minutes; after filtration from the unattacked substance, the carmine colcured solution was acidified with acetic acid, and the precipitate collected, and identified, by its characteristic sodium salt, in the manner above described.

The anhydride and orthotoluylenediamine interact very readily, a thoroughly typical azine being formed: 2 grams of the anhydride, 1 gram of orthotoluylenediamine hydrochloride, and 3 grams of crystallised sodium acetate are heated in 70 c.c. of acetic acid. Soon after the acid commences to boil, and before the anhydride has entirely passed into solution, the azine commences to separate in small clusters of microscopic needles. The conversion is complete in a few minutes, but, before the boiling is finally discontinued, it is well to 
ascertain, with the help of the microscope, that no unchanged crystals of the anbydride are present. The substance is then collected, washed with acetic acid or alcohol, and finally with water. For analysis, the azine was twice crystallised from benzene, in which, as in other ordinary organic solvents, it dissolves with considerable difficulty. It was thus obtained in orange-yellow, microscopic needles which can be heated to $245^{\circ}$ without undergoing change.

$0 \cdot 1701$ gave 8.5 c.c. moist nitrogen at $30^{\circ}$ and $764 \mathrm{~mm} . \quad \mathrm{N}=5 \cdot 43$. $0.2189,0.6516 \mathrm{CO}_{2}$ and $0.0815 \mathrm{H}_{2} \mathrm{O} . \quad \mathrm{C}=81 \cdot 17 ; \mathrm{H}=4.13$. $\mathrm{C}_{34} \mathrm{H}_{20} \mathrm{~N}_{2} \mathrm{O}_{3}$ requires $\mathrm{C}=80.95 ; \mathrm{H}=3.96 ; \mathrm{N}=5.55$ per cent.

The azine dissolves in concentrated sulphuric acid yielding a brownish-violet coloured solution, from which an orange coloured salt is precipitated on slight dilution. When moistened with concentrated hydrochloric acid, it becomes more deeply orange; and when heated with zinc-dust, and alcohol acidified with hydrochloric acid, a violet coloured solution is obtained, which readily absorbs oxygen, again becoming orange.

The azine was heated with orthotoluglenediamine, under varied conditions, but as no interaction occurred, we were unable to demonstrate the presence of a second orthoquinone group.

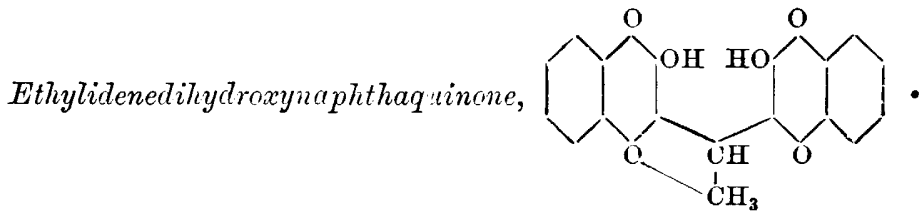

Schoch has already studied the action of acetaldehyde on hydroxynaphthaquinone (Diss., Marburg, 1888, p. 24), and obtained a product fusing at $154-157^{\circ}$, which refused to crystallise, and on analysis gave figures agreeing with the above formula. In spite of the analytical figures, the compound must have been in a very impure condition, as ethylidenedihydroxynaphthaquinone crystallises readily, and fuses at about $190^{\circ}$ when rapidly heated; its fusing point is not, however, quite constant.

After a number of preliminary experiments, we have sncceeded in satisfactorily preparing the compound in the following manner:5 grams of pulverised hydroxynaphthaquinone were heated under pressure in a steam bath (at about $100^{\circ}$ ) for five hours with 50 c.c. of alcohol and 50 c.c. of Schuchardt's "coucentrated aldehyde" (not absolute). After the heating had been in progress a short time, the bottle was slightly agitated, so as to ensure the complete dissolution of the hydroxynaphthaquinone. The new compound separated, as the liquid cooled, in a comparatirely pure condition, in small, heary crystals; 
33 grams were obtained. The mother liquor, on evaporation, yielded an additional quantity of the substance, but the condition of this was less satisfactory, and, in order to purify it, it was dissolved as completely as possible in a cold, aqueous 1 per cent. solution of sodium hydroxide. After fitration from the insoluble residue,* the substance was reprecipitated without unnecessary delay $\dagger$ by adding acetis acid, and was then collected, dried, and crystallised from alcohol.

Ethylidenedihydroxynaphthaquinone can be readily distinguished from hydroxynaphthaquinone by the carmine-red colour of its solution in dilute sodium hydroxide, which presents a sirong contrast to the orange-red solution of the latter.

The new compound was recrystallised from alcohol for analysis. It was obtained in irregular, heavy, golden-yellow crystals which melt when rapidly heated, but not perfectly sharply, at about $190^{\circ}$. The melting point is influenced to some extent by the manner of heating, and the fused compound gradually darkens and decomposes even as the temperature falls.

$$
\begin{gathered}
0.1920 \text { gave } 0.4950 \mathrm{CO}_{2} \text { and } 0.0655 \mathrm{H}_{2} \mathrm{O} . \quad \mathrm{C}=70.31 ; \mathrm{H}=3 \cdot 79 . \\
\mathrm{C}_{22} \mathrm{H}_{14} \mathrm{O}_{6} \text { requires } \mathrm{C}=70.58 ; \mathrm{H}=3 \cdot 74 \text { per cent. }
\end{gathered}
$$

$\alpha \beta-$ Anhydride of Ethylidenedihydroxynaphthaquinone.

Ethylidenedihydroxynaphthaquinone is readily and almost quantitatively converted into its pseudo-anhydride by the action of concentrated sulphuric acid. 2.5 grams of the compound were dissolved in 15 c.c. of concentrated sulphuric acid, and, after standing about 10 minutes, the solution was poured into a relatively large quantity of water. The orange precipitate was collected on a filter, and as soon as the acid had been mostly displaced by water, it was washed with a 1 per cent. solution of sodium hydroside to remove any unchanged ethylidenedihydroxynaphthaquinone, and then again with water. When dry, the compound was crystallised from acetic acid; it separated in orange needles, which lost some of their brilliancy of colour when exposed to diffused daylight.

Analysis gave the following figures.

0.1881 gave $0.5089 \mathrm{CO}_{2}$ and $0.0606 \mathrm{H}_{2} \mathrm{O} . \quad \mathrm{C}=73.78 ; \mathrm{H}=3.57$. $\mathrm{C}_{22} \mathrm{H}_{12} \mathrm{O}_{5 \ddagger} \ddagger$ requires $\mathrm{C}=74.15 ; \mathrm{H}=3.37$ per cent.

* This consists of a dark red compound which dissolves in concentrated sulphuric acid, affording a green solution, and probably corresponds with the red substance (compare footnote, p. 80 ) observed in the preparation of benzylidenedihydroxynaphthaquinone, which also gives a green solution when dissolred in sulphuric acid.

† Ethylidenedihydroxynaphthaquinone gradually undergoes change even in cold dilute alkaline solution.

¥ By heating paraldehyde with hydroxynaphthaquinone, Schoch obtained an on analysis approximate to those required by the formula $\mathrm{C}_{22} \mathrm{H}_{12} \mathrm{O}_{5}$ (Diss., Mar- 
When boiled with a dilute aqueous solution of sodium hydroxide. the anbydride gradually passes into solution, being reconverted into ethylidenedihydroxynaphthaquinone, which then rapidly undergoes change.

The anhydride interacts very readily with orthotoluylenediamine; the resulting compound was obtained in essentially the same manner as the corresponding azine from the $\beta$-anhydride of benzylidenedihydroxynaphthaquinone. Two preparations were made for analysis : the one (a) was crystallised from benzol; the other $(b)$ from chloroform.

(a) 0.1276 gave 6.9 c.c. moist nitrogen at $27^{\circ}$ and $762 \mathrm{~mm} . \mathrm{N}=5.98$. (b) $0.2216,11 \cdot 7,, \quad, \quad 24.5,770, \% \quad \mathrm{~N}=5.98$. (a) $0.1705,0.4917 \mathrm{CO}_{2}$ and $0.0728 \mathrm{H}_{2} \mathrm{O} . \quad \mathrm{C}=78.65 ; \mathrm{H}=4 \cdot 74$. (a) $0.1636,0.4744 \quad, \quad, 0.0669 \quad, \quad \mathrm{C}=79.08 ; \mathrm{H}=4.54$. (b) $0.1652,0.4743,, \quad, 0.0675,, \quad \mathrm{C}=7890 ; \mathrm{H}=4.51$. $\mathrm{C}_{29} \mathrm{H}_{18} \mathrm{~N}_{2} \mathrm{O}_{3}$ requires $\mathrm{C}=78.73 ; \mathrm{H}=4.07 ; \mathrm{N}=6.33$ per cent.

The compound crystallises in minute, orange, woolly needles which dissolve in concentrated sulphuric acid yielding a brownish-violet coloured solution, and in this, as in other respects, precisely resembles the azine already described from the $\beta$-anhydride of benzylidenedihydroxynaphthaquinone.

\section{aß-Anhydride of Amylidenedihydrox'ynaphthaquinone.}

A mixture of 3 grams of hydroxynaphthaquinone, 3 c.c. of KahIbaum's valeraldehyde, and 9 c.c. of alcohol was boiled for three hours, a reflux condenser being used. A drop of the solution was then diluted with alcohol, and mixed with a weak aqueous solution of sodium hydroxide; the intense crimson colour developed showed that the hydroxynaphthaquinone had undergone change, and, although the compoumd was not isolated, the solution undoubtedly contained amylidenedihydroxynaphthaquinone. ${ }^{*}$ To convert this into its $\alpha \beta$-anhydride, the solution was evaporated to dryness in a dish, on a water orange-coloured substance which he was unable to crystallise. The figures obtained burg, 1883, p. 26).

* In the hope of obtaining lapachol synthetically, I have studied the behaviour of hydroxynaphthaquinone in contact with raleraldehyde under raried conditions. and hare found that if these compounds are heated in acetic acid solution, in the presence of a sufficiently large quantity of hydrochloric acid, an interaction occurs entirely different from the abore. The resulting compound has been analysed by Mr. C. C. Burger, who has also prepared and analysed its acetyl derivatire. The figures obtained prore that under these conditions, interaction occurs in the sense of the equation $\mathrm{C}_{10} \mathrm{H}_{6} \mathrm{O}_{3}+\mathrm{C}_{4} \mathrm{H}_{9} \cdot \mathrm{COH}=\mathrm{C}_{15} \mathrm{H}_{14} \mathrm{O}_{3}+\mathrm{H}_{2} \mathrm{O}$.

The compound is isomeric with lapachol; it crystallises in brilliant orange-red reedles, melting at $119-120^{\circ}$, and with the alkalis forms riolet salts which crystallise very readily. The acetyl derivative fuses at $74^{\circ}$. 
bath, to drive off the excess of valeraldehyde, and the residue was dissolved in 20 c.c. of warm acetic acid, and transferred to a flask which was then immersed in ice-cold water. A mixture of 60 c.c. of concentrated sulpharic acid and 20 c.c. of acetic acid, also previously cooled, was then added, the flask being kept, during the whole operation, in water cooled by ice; after a few minutes, it was poured into a large volume of water, and the precipitate which formed was collected on a filter and thoroughly washed. When dry, it was twice crystallised from acetic acid and analysed.

$$
\begin{gathered}
0.1729 \text { gare } 0.4759 \mathrm{CO}_{2} \text { and } 0.0711 \mathrm{H}_{2} \mathrm{O} . \mathrm{C}=75.06 ; \mathrm{H}=4.56 . \\
\mathrm{C}_{25} \mathrm{H}_{15} \mathrm{O}_{5} \text { requires } \mathrm{C}=75.37 ; \mathrm{H}=4.52 \text { per cent. }
\end{gathered}
$$

The anhydride was obtained in small, orange needles (sometimes also in small, reddish-brown plates), which commence to darken at a temperature near to $200^{\circ}$, and fuse and decompose at a somewhat higher temperature.

The presence of an orthoquinone group was demonstrated by the behaviour of the componnd with orthotoluylenediamine, the azine being prepared essentially in the manuer previously described (p. 81) in the case of the corresponding benzylidene compound. For analysis, it was purified by crystallisation from benzene, in which it is much more soluble than either the corresponding benzylidene or ethylidene compounds. It was obtained in orange-coloured needles which gave precisely the same colour tests with sulphuric acid as the azines already described.

0.1618 gave $0.4705 \mathrm{CO}_{2}$ and $0.0753 \mathrm{H}_{2} \mathrm{O} . \quad \mathrm{C}=79.30 ; \mathrm{H}=5.17$.

$0.1653,8.20$ c.c. moist nitrogen at $26^{\circ}$ and $767 \mathrm{~mm} . \quad \mathrm{N}=5.55$. $\mathrm{C}_{32} \mathrm{H}_{24} \mathrm{~N}_{2} \mathrm{O}_{3}$ requires $\mathrm{C}=79.34 ; \mathrm{H}=4.95 ; \mathrm{N}=5.78$ per cent.

Cuminic, Salicylic, and Cinnamic Aldehydes.

These aldehydes and hydroxynaphthaquinone interact very readily (compare Schoch). By following the methods already given, we have succeeded in converting the resulting compounds, apparently quantitatively, into the $\alpha \beta$-anhydrides. In all three cases, the anhydrides proved to be bright red, crystalline componnds, which were readily acted on by orthotoluylenediamine, giving characteristic azines. The compounds were not analysed, and for that reason are not described more fully here.

I shall defer for a short time the discussion of the constitution of the compound and its bearing on that of lapachol; but I may state that $I$ have succeeded in converting it by a number of interesting changes into compounds containing the full number of carbon atoms present in lapachol, and which I have also prepared from lapachol itself. The behaviour of other aldehydes under s milar conditions will also be studied.-S. C. H. 